\title{
Estratégias pedagógicas na educação médica ante os desafios da Covid-19: uma revisão de escopo
}

\author{
Pedagogical strategies in medical education to the challenges of Covid-19: scoping review

\begin{tabular}{|c|c|}
\hline Rodrigo Otávio Moretti-Pires'1 & rodrigo.moretti@ufsc.br \\
\hline Dalvan Antônio de Campos ${ }^{1}$ & campos@gmail.com \\
\hline Zeno Carlos Tesser Junior ${ }^{1}$ & zenotjunior@gmail.com \\
\hline oão Batista de Oliveira Junior ${ }^{1}$ & jjeducauel@gmail.com \\
\hline Bárbara de Oliveira Turatti ${ }^{1}$ & barbara.olliveira@gmail.com \\
\hline Danie & ddaniel.canavese@gmail.c \\
\hline
\end{tabular}

\section{RESUMO}

Introdução: Os desafios à continuidade do processo ensino-aprendizagem universitário ante as medidas de combate à pandemia da Covid-19 tornaram mais importante o debate sobre o uso de tecnologias de informação e comunicação (TIC) no ensino médico. Diversas estratégias foram empregadas no mundo por docentes para a continuidade das atividades pedagógicas.

Objetivo: Este estudo teve como objetivo investigar as estratégias e os usos de TIC no ensino médico ante a pandemia de Covid-19.

Método: Examinaram-se sistematicamente cinco bases de dados, nas quais se empregaram as expressões e os termos "covid-19", "ensino médico", "educação superior" e "estudantes" em português, inglês e espanhol, o que resultou em 321 citações iniciais, com 18 referências finais após a aplicação de critérios de inclusão e exclusão.

Resultado: Quatro temas-chave foram identificados na literatura: 1.“Desafios para o ensino médico anteriores à Covid-19”; 2. “Desafios na migração para o ensino a distância"; 3. "Estratégias para a superação de desafios relacionadas ao ambiente de aprendizagem virtual"; e 4. “Estratégias para a superação de desafios relacionadas às avaliações".

Conclusão: No contexto da pandemia de Covid-19, o emprego de TIC no ensino médico se mostrou importantíssimo, pois se encontraram quatro estratégias, entre as quais se destacaram o aprimoramento em áreas em que as TIC já eram utilizadas, a migração de algumas áreas mais articuladas e experiências em disciplinas clínicas e procedurais. Também houve preocupação sobre os impactos do uso de TIC em substituição da presença de estudantes nos ambientes de aprendizagem médicos.

Palavras-chave: Covid-19; Ensino Superior; Educação Médica; Ensino a Distância; Scoping Review.

\section{ABSTRACT}

Introduction: The challenges brought by the continuity of the university teaching-learning process in the face of the measures to combat the pandemic of COVID-19 made the debate on the use of information and communication technologies (ICT) in medical education more important. Several strategies were used by teachers worldwide to continue their teaching activities.

Objective: to investigate the strategies and uses of ICT in medical education in the face of the COVID-19 pandemic.

Method: Five databases were systematically assessed, using the terms "COVID-19", "medical education", "higher education" and "students", in Portuguese, English and Spanish, resulting in 321 initial citations, with 18 final references after applying the inclusion and exclusion criteria.

Result: Four key topics were identified in the literature: (1) Challenges for Medical Education prior to COVID-19; (2) Challenges in migrating to remote education; (3) Strategies to overcome challenges related to the learning environment; and (4) Strategies to overcome challenges related to assessments and exams.

Conclusion: The use of ICT in medical education in the context of the COVID-19 pandemic showed to be especially important, with considerations regarding the improvement in areas that were already used, the migration of some more articulated areas and experiences in clinical and procedural disciplines. There was also concern about the impacts of using ICT to replace the in-person presence of students in medical learning environments.

Keywords: COVID-19; Higher Education; Medical Education; Distance Learning; Scoping Review.

1 Universidade Federal de Santa Catarina, Florianópolis, Santa Catarina, Brasil.

${ }^{2}$ Universidade Federal do Rio Grande do Sul, Porto Alegre, Rio Grande do Sul, Brasil.

Editora-chefe: Daniela Chiesa

Editor associado: Kristopherson Lustosa Augusto

Recebido em 17/08/20; Aceito em 09/12/20.

Avaliado pelo processo de double blind review. 


\section{INTRODUÇÃO}

Mudanças importantes em termos mundiais foram ocasionadas pelo novo coronavírus, o coronavírus da síndrome respiratória aguda grave 2 (severe acute respiratory syndrome coronavirus 2 - Sars-CoV-2). Em 11 de março de 2020, declarou-se pandemia da coronavirus disease 2019 (Covid-19) com 118 mil casos registrados e quatro mil óbitos ${ }^{1 .}$ Como o isolamento social é a mais importante estratégia empregada nessa emergência sanitária, houve mudanças em todos os campos sociais, incluindo as universidades e, nelas, o ensino médico. A pandemia de Covid-19 provocou disrupção nas rotinas hospitalares como um todo, nos serviços de saúde, nas escolas de Medicina e em outros importantes ambientes de aprendizagem, além da centralidade que os profissionais da saúde têm na sociedade para manutenção de vidas ${ }^{2}$. O cancelamento sistemático das aulas presenciais e a substituição por aulas mediadas por tecnologia a distância trouxeram grandes questionamentos em termos de educação médica para realização das disciplinas pré-clínicas e clínicas ${ }^{2,3}$.

O emprego de tecnologias de informação e comunicação (TIC) na educação médica não é uma novidade trazida pela Covid-19, pois elas já são utilizadas desde o final do século $X X^{4}$, com amplos estudos sobre protocolos próprios e debates pedagógicos dessas estratégias pedagógicas 5 . No entanto, não se trata de um caminho empregado em todos os setores do ensino médico, uma vez que o modelo presencial, centrado em conteúdos e no desenvolvimento de habilidades clínicas, ainda é preponderante ${ }^{5}$. Mesmo assim, existem experiências registradas na literatura sobre ensino médico mediado por TIC, inclusive em fases avançadas do curso como o internato médico ${ }^{6}$.

A presente revisão de escopo tem por objetivo investigar as estratégias de uso de TIC empregadas no ensino médico ante os desafios da pandemia de Covid-19.

\section{MÉTODO}

Realizou-se uma revisão de escopo na qual se utilizou manual do Joanna Briggs Institute ${ }^{7}$, que pressupõe a síntese de resultados e temáticas em desenvolvimento. $O$ foco foi mapear o que existe de relevante na literatura do campo de interesse, uma opção pertinente ante os desafios para a educação médica advindos da pandemia de Covid-19. A questão de pesquisa identificada foi a seguinte:

- Quais são as estratégias empregadas no ensino médico ante a pandemia de Covid-19?
Para sistematizar a escrita do trabalho, optou-se pela utilização das recomendações do PRISMA-SrC, um checklist com 21 itens específico para melhorar a qualidade das revisões de escopo ${ }^{8}$.

As buscas foram realizadas em português, espanhol e inglês, no período de 14 a 31 de julho de 2020. Utilizaramse quatro bases científicas: Scopus, PubMed, BVS e SciELO. No portal Google Scholar, realizou-se o mapeamento da literatura cinza. No Scopus, usaram-se as seguintes palavras-chave: (TITLE-ABS-KEY (covid-19) AND TITLE-ABSKEY ("medical education") AND TITLE-ABS-KEY (student) OR TITLE-ABS-KEY ("high education")) AND (LIMIT-TO (SUBJAREA, "MEDI")). Dois membros da equipe de pesquisa realizaram todas as etapas de maneira independente: busca, seleção por títulos, resumo e leitura na íntegra. Em caso de divergências, houve avaliação de um terceiro avaliador, que deliberava em caráter final sobre a inclusão ou não do artigo na etapa.

Adotaram-se os seguintes critérios de inclusão: enfoque na educação médica, apresentação de, pelo menos, uma estratégia empregada no ensino após o início da Covid-19, artigos originais, relatos de experiências e comentários baseados em iniciativas educacionais. Foram critérios de exclusão: artigos que se referiam à percepção de estudantes e/ou docentes sobre a pandemia, artigos sobre avaliação de preferências, estudos com aspectos clínicos sobre a Covid-19, estudos estritamente sobre biossegurança para os estudantes, considerações estritamente teóricas, estudos que não apresentavam claramente quais recursos foram empregados para lidar com os desafios da pandemia de Covid-19 e artigos apenas com considerações sem caminhos ou intervenções e estudos sobre saúde mental de professores e estudantes.

Para a análise dos artigos incluídos, realizou-se um mapeamento que consistiu no levantamento prévio dos temas-chave apresentados nos resultados dos artigos, no agrupamento dessas informações, na identificação de vínculos entre os pontos e na síntese dos estudos, com o propósito de classificar e reclassificar o material produzido de acordo com a pergunta da revisão de escopo.

\section{RESULTADO}

Nas buscas realizadas, obteve-se um total de 321 artigos, dos quais 18 foram incluídos para construção da revisão, após seleção pela equipe de pesquisa, conforme mostra a Figura 1. 
Figura 1. Fluxograma da revisão

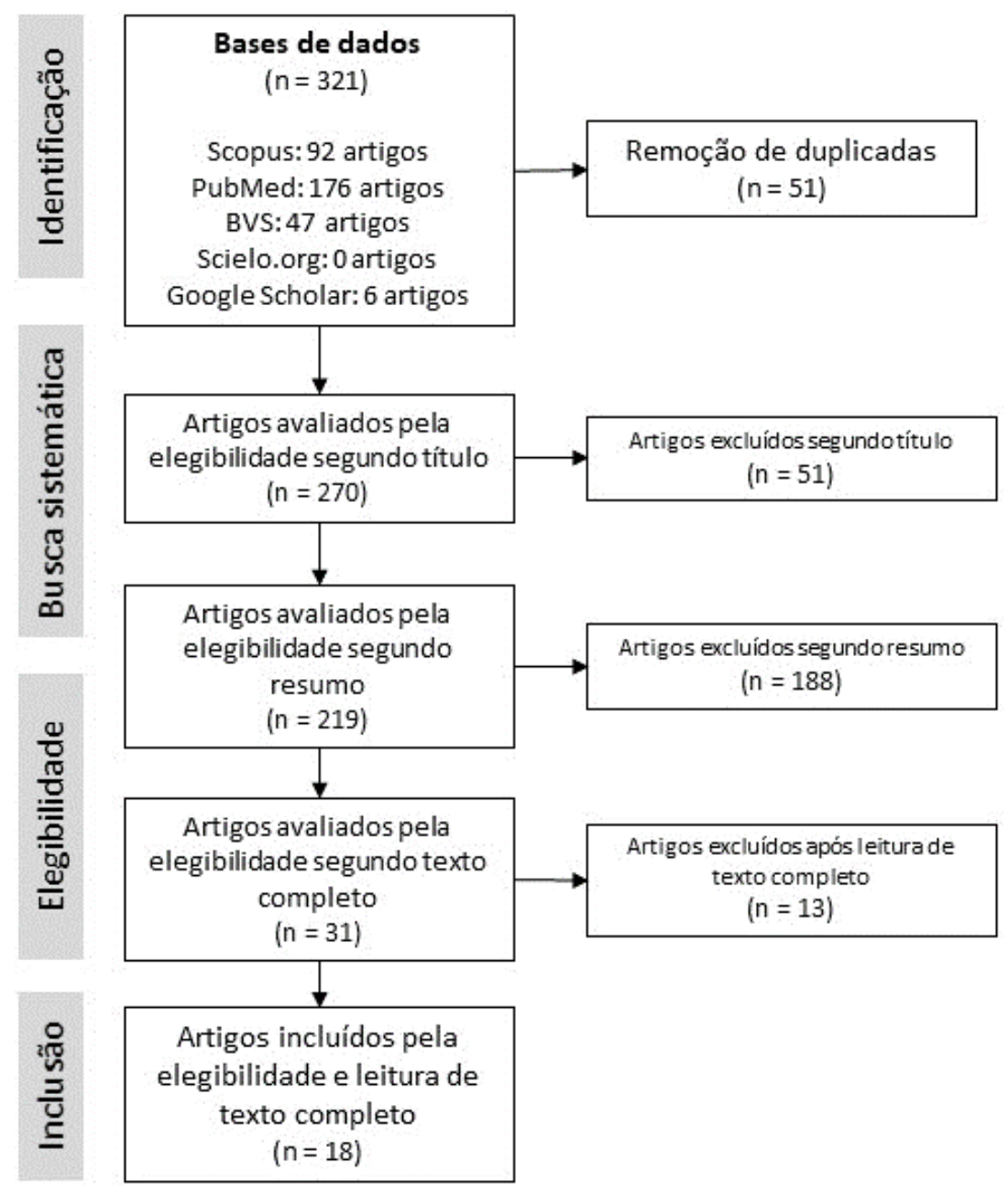

O Quadro 1 apresenta os dados bibliométricos dos estudos incluídos.

Quadro 1. Características bibliométricas dos estudos incluídos

\begin{tabular}{|c|c|c|c|c|c|c|}
\hline Autores & País & Palavras-chave & Tipo de artigo & Revista & Mês de publicação & Foco do artigo \\
\hline Birch et al. ${ }^{9}$ & $\begin{array}{l}\text { Reino } \\
\text { Unido }\end{array}$ & $\begin{array}{l}\text { Medical students, } \\
\text { Covid-19, medical } \\
\text { school examinations, } \\
\text { online examinations, } \\
\text { open-book examination. }\end{array}$ & Artigo original & $\begin{array}{l}\text { Medical } \\
\text { Education } \\
\text { Online }\end{array}$ & Junho de 2020 & $\begin{array}{l}\text { Mudanças no ensino e na } \\
\text { avaliação dos estudantes } \\
\text { de Medicina no King's } \\
\text { College London. }\end{array}$ \\
\hline $\begin{array}{l}\text { Mukhtar et } \\
\text { al. }^{10}\end{array}$ & Paquistão & $\begin{array}{l}\text { Covid-19, education, } \\
\text { medical, undergraduate, } \\
\text { online learning. }\end{array}$ & Artigo original & $\begin{array}{l}\text { Pakistan Journal } \\
\text { of Medical } \\
\text { Sciences }\end{array}$ & Maio de 2020 & $\begin{array}{c}\text { Recomendações sobre o } \\
\text { ensino a distância durante } \\
\text { a pandemia da Covid-19 } \\
\text { no Paquistão. }\end{array}$ \\
\hline Singal et al. ${ }^{11}$ & Índia & $\begin{array}{l}\text { Anatomy education, } \\
\text { body donation, } \\
\text { Covid-19, pandemic, } \\
\text { virtual classes. }\end{array}$ & Artigo original & Morphologie & Maio de 2020 & $\begin{array}{c}\text { Discutir os efeitos e as } \\
\text { saídas para os desafios } \\
\text { ao ensino de anatomia } \\
\text { durante a pandemia de } \\
\text { Covid-19. }\end{array}$ \\
\hline Chao et al. ${ }^{12}$ & $\begin{array}{l}\text { Estados } \\
\text { Unidos }\end{array}$ & $\begin{array}{c}\text { Virtual surgical } \\
\text { education, } \\
\text { undergraduate medical } \\
\text { education, Covid-19, } \\
\text { telemedicine, surgical } \\
\text { video capture. }\end{array}$ & Artigo original & $\begin{array}{l}\text { Journal of } \\
\text { Surgical } \\
\text { Education }\end{array}$ & Junho de 2020 & $\begin{array}{l}\text { Desenvolvimento de } \\
\text { atividades virtuais em } \\
\text { disciplina cirúrgicas. }\end{array}$ \\
\hline
\end{tabular}


Quadro 1. (Continuação) Características bibliométricas dos estudos incluídos

\begin{tabular}{|c|c|c|c|c|c|c|}
\hline Autores & País & Palavras-chave & Tipo de artigo & Revista & Mês de publicação & Foco do artigo \\
\hline Rosa et al. ${ }^{13}$ & Peru & $\begin{array}{l}\text { Educación médica, } \\
\text { educación a distancia, } \\
\text { infecciones por } \\
\text { coronavirus, Covid-19, } \\
\text { América Latina. }\end{array}$ & Artigo original & $\begin{array}{l}\text { Educación } \\
\text { Médica } \\
\text { Superior }\end{array}$ & Maio de 2020 & $\begin{array}{l}\text { Debate sobre as principais } \\
\text { formas de ensino a } \\
\text { distância nos cursos de } \\
\text { Medicina, no contexto da } \\
\text { Covid-19. }\end{array}$ \\
\hline Mishra et al. ${ }^{14}$ & $\begin{array}{l}\text { Estados } \\
\text { Unidos }\end{array}$ & $\begin{array}{c}\text { Coronavirus } \\
\text { disease-2019, } \\
\text { online education, } \\
\text { ophthalmology } \\
\text { education, virtual } \\
\text { curriculum. }\end{array}$ & Artigo original & Ophthalmology & Julho de 2020 & $\begin{array}{l}\text { Descrever a transição da } \\
\text { educação em oftalmologia } \\
\text { para um currículo virtual } \\
\text { durante a pandemia da } \\
\text { Covid-19. }\end{array}$ \\
\hline Gomez et al. ${ }^{15}$ & $\begin{array}{l}\text { Estados } \\
\text { Unidos }\end{array}$ & $\begin{array}{l}\text { Medical student } \\
\text { education, radiology } \\
\text { education, remote } \\
\text { learning, Covid-19. }\end{array}$ & Artigo original & $\begin{array}{l}\text { Academic } \\
\text { Radiology }\end{array}$ & Junho de 2020 & $\begin{array}{l}\text { Oferta de aulas virtuais de } \\
\text { diagnóstico de radiologia } \\
\text { em razão da pandemia da } \\
\text { Covid- } 19 .\end{array}$ \\
\hline $\begin{array}{l}\text { Krawiec et } \\
\text { al. }^{17}\end{array}$ & $\begin{array}{l}\text { Estados } \\
\text { Unidos }\end{array}$ & $\begin{array}{c}\text { Assessment in } \\
\text { health professions } \\
\text { education, Covid-19, } \\
\text { undergraduate medical } \\
\text { education. }\end{array}$ & Artigo original & Cureus & Junho de 2020 & $\begin{array}{l}\text { Módulos baseados em } \\
\text { casos virtuais para ensino } \\
\text { em estágio pediátrico no } \\
\text { contexto da pandemia da } \\
\text { Covid-19. }\end{array}$ \\
\hline Ko et al. ${ }^{18}$ & $\begin{array}{l}\text { Estados } \\
\text { Unidos }\end{array}$ & Não há. & $\begin{array}{l}\text { Relato de } \\
\text { experiência }\end{array}$ & $\begin{array}{c}\text { Journal } \\
\text { of Neuro- } \\
\text { Ophthalmology }\end{array}$ & Junho de 2020 & $\begin{array}{l}\text { Abordar a telessaúde } \\
\text { em neuro-oftalmologia, } \\
\text { incluindo desafios e } \\
\text { oportunidades atuais no } \\
\text { contexto da pandemia da } \\
\text { Covid-19. }\end{array}$ \\
\hline Finn et al. ${ }^{21}$ & $\begin{array}{l}\text { Reino } \\
\text { Unido }\end{array}$ & Não há & $\begin{array}{l}\text { Relato de } \\
\text { experiência }\end{array}$ & $\begin{array}{l}\text { Medical } \\
\text { Education }\end{array}$ & Maio de 2020 & $\begin{array}{c}\text { Estratégia educacional } \\
\text { empregando Twitter sobre } \\
\text { a Covid-19. }\end{array}$ \\
\hline Kumar et al. ${ }^{22}$ & Índia & $\begin{array}{l}\text { Dermatology practice } \\
\text { in shadow of Covid, } \\
\text { changing dermatology } \\
\text { practice post Covid. }\end{array}$ & $\begin{array}{c}\text { Comunicação } \\
\text { breve }\end{array}$ & $\begin{array}{c}\text { Dermatologic } \\
\text { Therapy }\end{array}$ & Abril de 2020 & $\begin{array}{c}\text { Atividades on-line para } \\
\text { ensino de dermatologia } \\
\text { no contexto da pandemia } \\
\text { da Covid-19. }\end{array}$ \\
\hline $\begin{array}{l}\text { Mathieson et } \\
\text { al. }^{23}\end{array}$ & $\begin{array}{l}\text { Reino } \\
\text { Unido }\end{array}$ & $\begin{array}{c}\text { Medical education, } \\
\text { assessment, open-book } \\
\text { examination, Covid-19. }\end{array}$ & $\begin{array}{c}\text { Comunicação } \\
\text { breve }\end{array}$ & $\begin{array}{l}\text { Medical } \\
\text { Education } \\
\text { Online }\end{array}$ & Junho de 2020 & $\begin{array}{l}\text { Exames médicos escritos } \\
\text { presencialmente para } \\
\text { a modalidade on-line } \\
\text { mediante o contexto da } \\
\text { pandemia de Covid-19. }\end{array}$ \\
\hline $\begin{array}{c}\text { Hofmann et } \\
\text { al. }^{24}\end{array}$ & $\begin{array}{l}\text { Estados } \\
\text { Unidos }\end{array}$ & Não há. & $\begin{array}{c}\text { Relato de } \\
\text { experiência }\end{array}$ & $\begin{array}{c}\text { Medical } \\
\text { Education }\end{array}$ & Maio de 2020 & $\begin{array}{c}\text { Adaptação de visitações } \\
\text { à beira do leito } \\
\text { usando recurso de } \\
\text { videoconferência durante } \\
\text { a pandemia da Covid-19. }\end{array}$ \\
\hline
\end{tabular}


Quadro 1. (Continuação) Características bibliométricas dos estudos incluídos

\begin{tabular}{|c|c|c|c|c|c|c|}
\hline Autores & País & Palavras-chave & Tipo de artigo & Revista & Mês de publicação & Foco do artigo \\
\hline $\begin{array}{l}\text { Chandra et } \\
\mathrm{al}^{25}\end{array}$ & $\begin{array}{l}\text { Estados } \\
\text { Unidos }\end{array}$ & Não há. & $\begin{array}{l}\text { Relato de } \\
\text { experiência }\end{array}$ & $\begin{array}{l}\text { Medical } \\
\text { Education }\end{array}$ & Junho de 2020 & $\begin{array}{l}\text { Chamadas clínicas } \\
\text { permitindo interação ao } \\
\text { vivo com os pacientes } \\
\text { e desenvolvimento de } \\
\text { habilidades interpessoais e } \\
\text { de comunicação. }\end{array}$ \\
\hline Rose $^{26}$ & $\begin{array}{l}\text { Estados } \\
\text { Unidos }\end{array}$ & Não há. & $\begin{array}{l}\text { Comunicação } \\
\text { breve }\end{array}$ & JAMA & Março de 2020 & $\begin{array}{l}\text { Descrever como a } \\
\text { Covid-19 pode afetar } \\
\text { os ambientes de } \\
\text { aprendizagem na } \\
\text { medicina e suas possíveis } \\
\text { implicações para o futuro } \\
\text { da educação médica. }\end{array}$ \\
\hline
\end{tabular}

Quatro temas-chave foram identificados na literatura: 1. "Desafios para o ensino médico anteriores à Covid-19"; 2. "Desafios na migração para o ensino a distância"; 3. "Estratégias para a superação de desafios relacionadas ao ambiente de aprendizagem virtual"; e 4. "Estratégias para a superação de desafios relacionadas às avaliações".

\section{Desafios para o ensino médico anteriores à Covid-19}

A maioria dos estudos incluídos apontou que a discussão sobre a forma de estruturação do currículo médico e sua centralização em um paradigma considerado pouco articulado com as revoluções das TIC já ocorria havia muito tempo, mas a questão se tornou mais contundente após a necessidade de migração ao on-line $e^{9,11}$. A centralidade do docente e do espaço clínico na formação e a posição pouco protagonista dos estudantes no processo ensino-aprendizagem também foram características marcadas nos artigos, a exemplo da dificuldade de migrar disciplinas avançadas nos cursos para outras formas que não a vivência em enfermarias e ambulatórios ${ }^{9}$. Um exemplo interessante refere-se a disciplina de Anatomia, considerada a "pedra angular" da formação médica, que, mesmo com recursos virtuais e simulações, ainda encontra grande resistência para abandonar o ensino com cadáveres ${ }^{11}$. O desempenho profissional dos médicos futuros é uma preocupação da comunidade acadêmica, haja vista o volume de materialidades e espaços necessários para a formação desse profissional, uma vez que, além dos conhecimentos anatômicos, existem desenvolvimentos de habilidades técnicas que têm pouca possibilidade de alternativas que não presenciais nas atividades didático-pedagógicas ${ }^{9,11}$.

A necessidade de migração para o ensino a distância tem gerado grande pressão tanto nos estudantes - preocupados com o desenvolvimento de habilidades - como nos educadores, que passam a forçosamente a se aventurarem território desconhecido, como é o caso da digitalização das salas de aulas ${ }^{14,15}$, a despeito do uso cada vez mais presente de recursos eletrônicos tanto na clínica médica geral como nos espaços educacionais ${ }^{17}$. Apesar da tecnologia avançada e potente nas universidades, incluindo hardware e software, ainda há necessidade de avanços significativos para permitir um aprendizado on-line eficaz ${ }^{10}$, além de uma mudança de paradigma educacional que permita o protagonismo do estudante, que ainda é muito dependente da sala de aula e da tutela docente ${ }^{10}$.

Nesse sentido, deve-se ressaltar o fato muito comum de as pessoas procurarem informações sobre saúde em plataformas como o YouTube, frequentemente usado como fonte de educação para maiores conhecimentos sobre as doenças ${ }^{19}$. Ao mesmo tempo, existem, há alguns anos, avanços em áreas como a teledermatologia, alternativa prontamente viável como medida para resolução de alguns aspectos das disciplinas presenciais do campo ${ }^{22}$.

\section{Desafios na migração para o ensino a distância}

Uma questão importantíssima levantada trata sobre possibilidades e impossibilidades de migração para o virtual, ao menos de maneira mais imediata, com uma importante divisão destacada entre as disciplinas pré-clínicas, as disciplinas clínicoteóricas e o internato ${ }^{10,12,13}$. Outrossim, apenas aquelas primeiras e com foco teórico teriam mais vocação para a migração para o espaço virtual com velocidade ${ }^{10}$, uma vez que diversas habilidades próprias do fazer clínico não são conseguidas na modalidade virtual. Isso parece estar também relacionado ao próprio ato clínico, que demanda presença tanto do profissional como do paciente, para além da educação médica em si ${ }^{11}$.

Há limitações imensas para as vivências clínicas e de ambiente cirúrgico, além das visitas ao consultório, assim como a impossibilidade de algumas especialidades cirúrgicas serem migradas para o ensino a distância ${ }^{12}$. Enquanto as disciplinas pré-clínicas apresentam maior facilidade por causa da menor interação com o paciente, do melhor acesso ao material 
educacional virtual, do uso de plataformas e da aprendizagem baseada em problemas, as clínicas têm possibilidade de migração, desde que se utilizem simuladores de realidade virtual e pacientes simulados ${ }^{13}$, guardadas as devidas restrições tanto na construção de conhecimento clínico quanto na aquisição de habilidades ${ }^{14,15,20}$.

Em termos das TIC desenvolvidas e empregadas, existem grandes desafios em ambientes com restrições de recursos, incluindo falta de disponibilidade generalizada, diagnóstico incorreto resultante de baixa qualidade das fotos, dados médicos on-line inadequados do paciente, lacuna de comunicação entre o médico e os pacientes em tratamento e problemas inerentes à realização de investigações, o que pode gerar prejuízo educacional irreparável22.

\section{Estratégias para a superação de desafios relacionadas ao ambiente de aprendizagem virtual}

Em relação às estratégias empregadas para as atividades didático-pedagógicas, a literatura aponta para as plataformas de videoconferência que foram anteriormente muito utilizadas na educação e na telemedicina ${ }^{10}$. Com a pandemia, houve a necessidade de diversificar a utilização, com no caso de encontros virtuais formados conforme ensino baseado em problemas ${ }^{10,12,13}$. Essas plataformas têm grande diversificação das modalidades de ensino a distância por meio do uso de $\mathrm{TIC}^{13}$. Há forte recomendação de ensino em pequenos grupos, o que facilita a interatividade ${ }^{13,14,26}$. É preconizado o uso da sala de aula invertida, como estratégia pedagógica, com o corpo docente mediando os conteúdos e as informações que devem ser acessados e estudados pelos discentes antes da aula ${ }^{26}$.

Em termos das plataformas para ambiente virtual de aprendizagem, citaram-se Microsoft Teams, Google Meet, Edmodo, Moodle e Blackboard ${ }^{10,15}$; no caso das plataformas de videoconferência, mencionaram-se Zoom, Skype para empresas, WebEx e Adobe Connect ${ }^{10,14,15}$. Também o Twitter foi empregado como espaço para interação entre estudantes e corpo docente, especialmente para resolução de dúvidas e problemas médicos, com ampla interação ${ }^{15,20}$. Registraram-se ainda os seguintes procedimentos: pré-gravação das aulas, uso de chat assíncrono e mesmo marcação de horários síncronos para supervisão e suporte educacional-pedagógico ${ }^{15}$.

Em relação às estratégias pedagógicas para as atividades virtuais, destacam-se o emprego de mapa mental do exame, diagnóstico diferencial e resumo do gerenciamento, com links para recursos a serem empregados em casos clínicos16; fóruns de discussão assíncronos ${ }^{16}$; um simpósio que facilita as interações sociais e a presença do professor $^{16}$; portfólio de aprendizado que facilita os aspectos dos objetivos pessoais e reflete o domínio organizacional ${ }^{16}$; apresentação virtual de casos pelos próprios estudantes ${ }^{17}$; rodadas de discussão virtuais ${ }^{17}$; e suporte aos estudantes por meio de acompanhamentos síncrono e assíncrono nas mídias sociais por especialistas ${ }^{18,21}$. Os seminários on-line e por videoconferência baseados na solução de problemas, geralmente acompanhados de resultados de investigação ou de um longo diálogo com o paciente, também foram relatados como estratégias ${ }^{22,23}$.

Além das disciplinas com maior carga horária teórica, as plataformas on-line também estão sendo empregadas para algumas atividades clínicas, como o Zoom para visitação no leito ${ }^{17}$ e outras fontes eletrônicas de contato com contato com o paciente, com o propósito de reduzir os impactos no desenvolvimento de habilidades clínicas ${ }^{17}$. Mensagens instantâneas para serviços de saúde virtuais, mensagens públicas sobre modificação comportamental, rastreamento epidemiológico e acesso a provedores virtuais de saúde já eram muito empregados ${ }^{18}$.

Encontraram-se experiências de dissecção virtual ${ }^{11}$ e uso de banco de imagens de anatomia ${ }^{11}$, patologia ${ }^{12}$ e radiologia ${ }^{15}$. Também a endoscopia, laparoscopia e cirurgia robótica são amplamente usadas em muitas disciplinas cirúrgicas e permitem a visualização cirúrgica por quem não está participando diretamente $\mathrm{e}^{12,14}$. Outra interessante estratégia foi a cirurgia transmitida virtual com câmera GoPro ${ }^{\circledR}$ e comunicação audiovisual bidirecional em tempo real entre o aluno e a equipe operacional ${ }^{12}$. Em outra experiência, aplicativos móveis validados para componentes do exame neuro-oftálmico foram empregados no ensino de oftalmologia ${ }^{18}$. Reforça-se a necessidade de que o corpo docente receba suporte para conduzir o ensino e a preceptoria clínica virtual ${ }^{14}$.

É possível avaliar, gerenciar e atender pacientes por meio da captura de vídeo e transmissão segura durante os procedimentos e para a prestação de cuidados em um ambiente de telessaúde ${ }^{12}$, da mesma forma que foram empregadas sessões de revisão cirúrgica de vídeo com preceptores ${ }^{14} \mathrm{e}$ sessões de casos clínicos mediadas por plataformas on-line por meio de oficinas remotas interativas e sessões de $\operatorname{casos}^{15}$.

Existem experiências de visitas de médico que afixou um iPad Pro e executou o aplicativo de videoconferência ${ }^{24}$; assim como o contato dos estudantes com os pacientes por meio do Zoom ${ }^{25}$ em sessões de laboratório, simulações e sessões de ultrassonografia à beira do leito, e também para instruções clínicas com pacientes padronizados e em ambientes autênticos de atendimento a eles ${ }^{26}$.

\section{Estratégias para a superação de desafios relacionadas às avaliações}

Uma preocupação muito marcada na literatura se referiu à avaliação do processo ensino-aprendizagem, já que tradicionalmente se privilegiam a memorização e as provas 
com enfoque conteudista. Além disso, por causa da pandemia, os exames passaram para as plataformas on-line $e^{10,26}$, o que demandou mudança de abordagem ${ }^{22,23}$.

Várias estratégias interessantes de avaliações on-line foram encontradas, incluindo avaliação oral por meio de videoconferência e comunicação próxima com os alunos ${ }^{12}$, apresentações orais ${ }^{12}$, avaliações assíncronas com consulta à bibliografia ${ }^{15}$, gravações em vídeo de apresentações sobre caso clínico ${ }^{15,18}$, exercícios de medicina baseada em evidências ao avaliar tratamentos ${ }^{14}$, exames com consulta ${ }^{23}$ e perguntas aleatorizadas no ambiente virtual de aprendizagem com tempo máximo estabelecido para conclusão ${ }^{23}$.

Em disciplinas que preveem o desenvolvimento de habilidades clínicas, houve consideráveis avanços para permitir a migração para os ambientes virtuais de aprendizagem, como o uso de relatório eletrônico no qual o supervisor clínico avalia as competências dos estudantes ${ }^{16,19}$; emprego de instrumento para avalição de habilidades clínicas ${ }^{15}$, fórum de discussão on-line e apresentação baseada em casos, avaliando a reflexão crítica e o uso da literatura ${ }^{16}$; construção de casos interativos simulados, que podem ser concluídos de forma assíncrona ${ }^{16,18}$; mapas mentais com links para documentos importantes, podcasts, vídeos e outros recursos; e uma seção final para autorreflexão crítica e ligação aos cenários clínicos ${ }^{16}$. Uma atividade que se destacou foi a solicitação aos estudantes que avaliassem a qualidade da informação em vídeos do YouTube relação ao que existe em termos de medicina baseada em evidências ${ }^{19}$.

De maneira mais integrada com a prática clínica, encontraram-se também exames práticos realizados em videoconferência, em que pacientes reais foram sendo substituídos por cenários e imagens de casos virtuais ${ }^{22}$. Em outro estudo, os preceptores do corpo docente supervisionaram e ouviram a conversação mediadas pelo Zoom entre estudantes e pacientes, forneceram feedback e comentários por meio da função de bate-papo do software, em tempo real, e intervieram quando necessário. Após a chamada, os alunos ajudaram a documentar esses retornos de chamada. Os professores preceptores avaliaram o desempenho dos alunos com as mesmas ferramentas de avaliação do estágio tradicional ${ }^{24}$.

\section{DISCUSSÃO}

O afastamento de alunos de estágios clínicos pode ter implicações significativas para o planejamento futuro da força de trabalho ${ }^{27}$. No entanto, a pandemia de Covid-19 tornou mais evidentes questões que já eram debatidas no ensino médico, tais como o papel do discente, o foco na formação por meio da vivência clínica e os desafios gerados por um modelo tradicional - como é o médico - perante o mundo contemporâneo e os avanços tecnológicos. Também a centralidade do estudante e não dos docentes no processo ensino-aprendizagem se tornou mais nítida como questão importante para as decisões pedagógicas dos cursos médicos ${ }^{28}$.

Ao mesmo tempo, os artigos apontam que existem grandes diferenças nas disciplinas de caráter geral das ciências da saúde, as específicas pré-clínicas médicas e as clínicas médicas, direcionando ao entendimento que deverão ser tomadas medidas diferentes, pensadas em possibilidades de mudança integral para o uso de TIC, mixagem, diminuição de carga presencial e até mesmo adiamento, caso seja significativa para a formação, com vistas a cinco possibilidades de abordagem: continuar, adiar, adaptar, descartar, adicionar outras formas ${ }^{29}$.

É potencial o uso de videoconferências para compensar a retirada das aulas clínicas realizadas diariamente. Uma webcam e um microfone passam a ser importantes para o trabalho em sala de aula ${ }^{30}$. O aprendizado misto, definido como a combinação do aprendizado presencial convencional e do ensino assíncrono ou síncrono, cresceu rapidamente e agora é amplamente utilizado no ensino médico ${ }^{30}$. Há também o emprego de webinários, fóruns de discussão, clubes de estudos clínicos, mídias sociais em geral e outras formas que possibilitem a interação entre os estudantes, professores e experts médicos ${ }^{31}$.

Questões referentes à capacitação do corpo docente para o uso das TIC e ao desenvolvimento de recursos de infraestrutura são defendidas pela literatura há muito tempo e se tornaram mais importantes ainda no contexto da pandemia de Covid-19. Ao mesmo tempo, diversas estratégias para a superação de desafios relacionadas ao ambiente de aprendizagem, às avaliações e aos exames foram adotadas de maneira interessante pelos docentes nos artigos incluídos no presente estudo, trazendo inspirações para a implementação imediata e o desenvolvimento futuro.

EmrazãodaemergênciadaCovid-19comoacometimento amplo na sociedade e que exigiu a reconfiguração do ensino no mundo todo, o presente estudo tem como potência a apresentação do que foi possível ser publicado na literatura científica internacional a respeito das estratégias empregadas para a continuidade do ensino médico perante as limitações da pandemia. No entanto, são evidentes as suas limitações, uma vez que o impacto da Covid-19 é recente, não havendo tempo para que todas as experiências nesse campo estejam na literatura, inclusive pelo tempo necessário para estudos mais robustos serem desenvolvidos, sintetizados na forma de publicação científica, submetidos, apreciados e aprovados por pares, além do processo de publicação nos veículos de divulgação científica. De todo modo, os achados da presente revisão de escopo são importantes como norte para futuras ações e adaptações em termos de educação médica durante a pandemia de Covid-19. Aos estudos futuros caberá avaliar 
a possibilidade de essas estratégias serem modificadas ou mesmo verificar se outras aparecerão conforme os cursos médicos se defrontam com os desafios trazidos pelo contexto sanitário e educacional.

\section{CONCLUSÃO}

Nesta revisão do escopo, as experiências relatadas de ensino médico em época de pandemia de Covid-19 sugerem a necessidade de adaptação da formação focada na presença do estudante em ambientes clínico-laboratoriais para uma situação mediada por TIC. Nesse sentido, os antigos desafios da educação médica ante as mudanças do mundo contemporâneo, como a teleconsulta, passaram a ser ressignificados como possibilidades de certa aprendizagem, visando minimizar os prejuízos oriundos do distanciamento social exigido como medida sanitária. Também o papel do estudante no processo ensino aprendizagem deve ser repensado, com certa centralidade na autonomia dadas as características do ensino remoto. De certa forma, pode-se concluir que as estratégias educacionais empregadas no ensino médico perante a pandemia de Covid-19 relacionam-se a quatro caminhos possíveis:

- Manter as estratégias pedagógicas já trabalhadas via on-line, como as atividades e classes previamente mediadas por tecnologia.

- Adaptar aulas, exercícios e simulações clínicas que eram presenciais e que se tornaram possíveis pela mediação de tecnologia on-line.

- Adaptar visitas e consultas clínicas com estratégias mistas em que um profissional faz a parte presencial com os pacientes e transmite (e às vezes interage) para os estudantes por tecnologia on-line.

- Adiar para o futuro elementos que são insubstituíveis - tanto clínicos como em procedimentos - que tem aspecto prático e de contato, além da questão da humanização

Em alguma medida, existem saídas possíveis para áreas mais clínicas e de contato com casos e procedimentos médicos, mas há o forte e importante questionamento sobre até que ponto essas adaptações podem ser empregadas sem prejuízo ao desenvolvimento de habilidades e competências aos futuros profissionais médicos.

\section{CONTRIBUIÇÃO DOS AUTORES}

Rodrigo Otávio Moretti-Pires e Dalvan Antônio de Campos conceberam a revisão e desenvolveram todas as etapas, desde a busca bibliográfica até a seleção progressiva de artigos, assim como a elaboração da primeira versão do manuscrito.
Zeno Carlos Tesser Junior atuou na seleção quando havia divergências. João Batista de Oliveira Junior, Bárbara de Oliveira Turatti e Daniel Canavese de Oliveira revisaram os resultados. Todos os autores contribuíram para a versão submetida.

\section{CONFLITO DE INTERESSES}

Os autores declaram não haver conflito de interesses neste estudo.

\section{FINANCIAMENTO}

Declaramos que não houve financiamento para a realização desta pesquisa.

\section{REFERENCES}

1. Antunes BBP, Peres IT, Baião FA, Ranzani OT, Bastos LSL, Silva $A A B$, et al. Progressão dos casos confirmados de COVID-19 após implantação de medidas de controle. Rev Bras Ter Intensiva. 2020;32(2):213-23.

2. Ferrel MN, Ryan JJ. The impact of Covid-19 on medical education. Cureus. 2020;12(3):e7492.

3. Diokno AC, Devries JM. The impact of Covid-19 on urologic practice, medical education, and training. Int Urol Nephrol. 2020;52(7):1195-8.

4. Chao LW, Silveira PS, Böhm GM. Telemedicine and education in Brazil. J Telemed Telecare. 1999;5:137-8.

5. Ellaway R, Masters K. AMEE Guide 32: e-learning in medical education Part 1: learning, teaching and assessment. Med Teach. 2008;30:455-73.

6. Lau FA, Mendes VF, Ventura AA, Bollela VR, Teixeira LAS. Implantação de estratégias de ensino à distância durante o internato: desafios e perspectivas. Rev Bras Educ Med. 2017; 41(2):269-77.

7. Peters MDJ, Godfrey C, McInerney P, Munn Z, Tricco AC, Khalil, H. Scoping reviews. In: Aromataris $\mathrm{E}$, Munn $Z$, editors. JBI manual for evidence synthesis. Adelaide: JBI, 2020.

8. Tricco AC, Lillie E, Zarin W, O'Brien KK, Colquhoun H, Levac D, Hempel S. PRISMA extension for scoping reviews (PRISMA-SCR): checklist and explanation. Ann Intern Med. 2018;169(7):467-73.

9. Birch $E$, de Wolf $M$. A novel approach to medical school examinations during the Covid-19 pandemic. Med Educ Online. 2020;25(1):1785680.

10. Mukhtar K, Javed K, Arooj M, Sethi A. Advantages, limitations and recommendations for online learning during Covid-19 pandemic era. Pak J Med Sci. 2020;36(COVID19-S4):S27-S31.

11. Singal A, Bansal A, Chaudhary P. Cadaverless anatomy: darkness in the times of pandemic Covid-19. Morphologie. 2020;104(346):147-50.

12. Chao TN, Frost AS, Brody RM, Byrnes YM, Cannady SB, Luu NN, et al. Creation of an interactive virtual surgical rotation for undergraduate medical education during the Covid-19 pandemic. J Surg Educ. 2021 January-February; 78(1): 346-350.

13. de la Rosa EV, Tam RV, Vargas MA, Saavedra LC, Olortegui JG. Educación médica a distancia en tiempos de COVID-19. Educ Med Super. 2020;34(2):e1.

14. Mishra K, Boland MV, Woreta FA. Incorporating a virtual curriculum into ophthalmology education in the coronavirus disease-2019 era. Curr Opin Ophthalmol. 2020;31(5):380-5.

15. Gomez E, Azadi J, Magid D. Innovation born in isolation: rapid transformation of an in-person medical student radiology elective to a remote learning experience during the Covid-19 pandemic. Acad Radiol. 2020 Sep; 27(9): 1285-1290.

16. Roskvist R, Eggleton K, Goodyear-Smith F. Provision of e-learning programmes to replace undergraduate medical students' clinical general practice attachments during Covid-19 stand-down. Educ Prim Care. 2020:1-8. 
17. Krawiec C, Myers A. Remote assessment of video-recorded oral presentations centered on a virtual case-based module: a Covid-19 feasibility study. Cureus. 2020;12(6):e8726.

18. Ko M, Busis NA. Tele-neuro-ophthalmology: vision for $20 / 20$ and beyond. J Neuro-Ophthalmol. 2020 Sep;40(3):378-384. doi: 10.1097/ WNO.0000000000001038.

19. Szmuda T, Syed MT, Singh A, Ali S, Özdemir C, Słoniewski P. YouTube as a source of patient information for coronavirus disease (Covid-19): a content-quality and audience engagement analysis. Rev Med Virol. 2020:e2132.

20. Huddart D, Hirniak J, Sethi R, Hayer G, Dibblin C, Rao BM et al. \#MedStudentCovid: how social media is supporting students during Covid-19. Med Educ. 2020. Oct;54(10):951-952.

21. Finn GM, Brown MEL, Laughey W, Dueñas A. \#pandemicpedagogy: using Twitter for knowledge exchange. Med Educ. 2020;54(2): 1190-1. doi: $10.1111 /$ medu. 14242

22. Kumar S, Bishnoi A, Vinay K. Changing paradigms of dermatology practice in developing nations in the shadow of Covid-19: lessons learnt from the pandemic. Dermatol Ther. 2020:e13472.

23. Mathieson $\mathrm{G}$, Sutthakorn $\mathrm{R}$, Thomas $\mathrm{O}$. Could the future of medical school examinations be open-book - a medical student's perspective? Med Educ Online. 2020;25(1):1787308.
24. Hofmann $\mathrm{H}$, Harding $\mathrm{C}$, Youm J, Wiechmann W. Virtual bedside teaching rounds with patients with Covid-19. Med Educ. 2020 May 13;54(1):959-60. doi: $10.1111 /$ medu. 14223 .

25. Chandra S, Laoteppitaks C, Mingioni N, Papanagnou. Zooming-out Covid: virtual clinical experiences in an emergency medicine clerkship. Med Educ. 2020 Dec;54(12):1182-1183.

26. Rose S. Medical student education in the time of Covid-19. JAMA. 2020;323(21);2131-2. doi: 10.1001/jama.2020.5227.

27. Halbert JA, Jones A, Ramsey LP. Clinical placements for medical students in the time of Covid-19. Med J Aust. 2020;213(2):69-69.e1.

28. Henry JA, Black S, Gowell M, Morris E; general practitioner. Covid-19: how to use your time when clinical placements are postponed. BMJ. May 2020 4;369:m1489.

29. Tolsgaard MG, Cleland J, Wilkinson T, Ellaway RH. How we make choices and sacrifices in medical education during the Covid-19 pandemic. Med Teach. 2020;42(7):741-3.

30. Moszkowicz D, Duboc H, Dubertret C, Roux D, Bretagnol F. Daily medical education for confined students during coronavirus disease 2019 pandemic: a simple videoconference solution. Clin Anat. 2020;33(6):9278. doi: 10.1002/ca.23601.

31. Schneider SL, Council ML. Distance learning in the era of Covid-19. Arch Dermatol Res. 2020 May 8:1-2. 